\title{
Moderate calorie restriction ameliorates reproduction via attenuating oxidative stress-induced apoptosis through SIRT1 signaling in obese mice
}

\author{
Shaohong Zhang ${ }^{1,2 \#}$, Mengxiao Zhang ${ }^{1,2 \#}$, Shuoshuo Sun ${ }^{1,2}$, Xiao Wei ${ }^{1,2}$, Yu Chen ${ }^{1,2}$, Peng Zhou ${ }^{1,2}$, \\ Rendong Zheng ${ }^{1,2}$, Guofang Chen ${ }^{1,2}$, Chao Liu ${ }^{1,2}$ \\ ${ }^{1}$ Endocrinology Department, Affiliated Hospital of Integrated Traditional Chinese and Western Medicine, Nanjing University of Chinese Medicine, \\ Nanjing, China; 'Jiangsu Province Academy of Traditional Chinese Medicine, Nanjing, China \\ Contributions: (I) Conception and design: S Zhang; (II) Administrative support: C Liu, G Chen; (III) Provision of study materials or patients: R \\ Zheng, Y Chen; (IV) Collection and assembly of data: P Zhou, X Wei; (V) Data analysis and interpretation: M Zhang, S Sun; (VI) Manuscript \\ writing: All authors; (VII) Final approval of manuscript: All authors. \\ \#These authors contributed equally to this work. \\ Correspondence to: Guofang Chen; Chao Liu. Endocrinology Department, Affiliated Hospital of Integrated Traditional Chinese and Western \\ Medicine, Nanjing University of Chinese Medicine, Nanjing, China; Jiangsu Province Academy of Traditional Chinese Medicine, Nanjing, China. \\ Email: chenguofang9801@163.com; profliuchao@163.com.
}

Background: Obesity is a growing global public health problem. It has been associated with diabetes, cardiovascular disease, cancer, and an increased risk of all-cause mortality, as well as infertility. Calorie restriction (CR) is an effective life intervention to defend against obesity. This study aimed to investigate the effects of long-term moderate CR on the reproductive function and underlying mechanisms in a mouse model of obesity.

Methods: Male C57BL/6 mice were randomized to two groups receiving either a standard diet (STD) or a high-fat diet (HFD) for 8 weeks to induce obesity. The HFD-induced obesity mice were further randomized into two groups: HFD group and CR group (reduced the mean amount of HFD by $25 \%$ ). After 12 weeks, the body weight, testicular coefficients, fasting blood glucose (FBG), serum triglyceride (TG), and total cholesterol (TC) were detected and measured. The sperm quality was detected by an automatic sperm quality analyzer (SQA-V). The structure of testicular tissues was examined by hematoxylin and eosin (HE) staining. Testicular cell apoptosis was assessed by terminal deoxynucleotidyl transferase dUTP nick end labeling (TUNEL) staining. The levels of NAD-dependent deacetylase sirtuin-1 (SIRT1) and antioxidative enzymes were detected in the testes.

Results: CR treatment reduced weight gain and increased testicle coefficients in HFD-induced obese mice. CR reduced the serum level of FBG, TG, and TC, and increased the serum levels of testosterone. Moreover, CR increased sperm count and motility, and sperm normality in obese mice. Furthermore, CR ameliorated the testicular morphological damage and cell apoptosis in obese mice. CR also attenuated the oxidative stress level and increased the protein expressions of SIRT1 in testicular tissues of obese mice.

Conclusions: Long-term moderate CR improves obese male fertility, probably by alleviating oxidative stress via activation of SIRT1 signaling.

Keywords: Calorie restriction (CR); high-fat diet (HFD); reproduction; oxidative stress; apoptosis

Submitted Apr 20, 2021. Accepted for publication Jun 02, 2021.

doi: $10.21037 / \mathrm{atm}-21-2458$

View this article at: http://dx.doi.org/10.21037/atm-21-2458 


\section{Introduction}

At present, with changes in diet and lifestyle, obesity epidemics have become an alarming public health problem over the past few decades. Obesity has been associated with diabetes, cardiovascular disease, cancer, and a range of musculoskeletal diseases $(1,2)$. Furthermore, it is gradually being recognized that unhealthy obesity leads to an increased risk of male infertility (3). Previous studies have suggested negative impacts of high body mass index on sex hormones and semen quality in men (4,5). Additionally, animal studies have also shown that high-fat diet (HFD) feeding induces reproductive dysfunction, such as a disrupted testicular structure, testicular germ cell apoptosis, and poor spermatogenesis $(6,7)$.

It is well known that obesity is caused by caloric overintake. Excessive fat intake contributes to a myriad of cellular dysfunctions, and finally trigger apoptotic cell death, a process termed lipotoxicity. Moreover, lipotoxic effects are mediated by oxidative stress (3). Oxidative stress has adverse effects on the structural and functional integrity of sperm (8). A 3-year multi-center study showed that obese men had an increased rate of sperm DNA damage, which may be associated with oxidative stress (9). Obesity-induced testicular oxidative stress and inflammatory response decreased testosterone synthesis and sperm quality in mice (10).

NAD-dependent deacetylase sirtuin-1 (SIRT1), which is an important regulator of longevity, regulates the level of oxidative stress (11). Furthermore, the catalytic activity of SIRT1 is essential for male fertility in mice (12). Therefore, regulating SIRT1 and oxidative stress levels may be an important method for the treatment of obesity-induced reproductive dysfunction. Calorie restriction (CR) is an intervention that reduces energy intake, but maintains adequate nutrition. As a "green and natural" therapy, CR has a significant effect on weight loss, which can alleviate a variety of diseases related to metabolic problems such as obesity, type 2 diabetes, cardiovascular disease, and cancer (13). It has been known that fasting can improve the reproductive and mental health in women (14). In terms of male fertility, weight-loss interventions result in an improvement of semen quality in obese men $(15,16)$. It has also been reported that CR increases the level of serum testosterone concentrations in obese men (17). However, the mechanisms underlying CR-mediated improvement of reproductive function has not yet been elucidated.

Therefore, this study was performed to evaluate the effects of long-term moderate CR on metabolic and reproductive damage in obese mice. We hypothesized that long-term moderate CR would improve the reproductive function by suppressing lipotoxicity in testicular tissue and oxidative stress via SIRT1 signaling.

We present the following article in accordance with the ARRIVE reporting checklist (available at http://dx.doi. org/10.21037/atm-21-2458).

\section{Methods}

\section{Reagents and antibodies}

Anti-SIRT1 antibody was purchased from Cell Signaling Technology (USA). Anti- $\beta$-actin antibody was purchased from Sigma (USA). Rodent chow was supplied by the Trophic Animal Feed High-Tech Co., Ltd. (China). The testosterone kit was purchased from R\&D system (USA). The insulin kit was purchased from the American Laboratory Products Company (USA). The superoxide dismutase (SOD), glutathione peroxidase (GSH-PX), and malondialdehyde (MDA) kits were purchased from Nanjing Jiancheng Bioengineering Inc. (China). The radio immunoprecipitation assay (RIPA) lysis buffer and bicinchoninic acid (BCA) kits were purchased from Beyotime Biotechnology Co., Ltd. (China).

\section{Animals and treatment}

All animal studies were approved by the Animal Experimentation Ethics Committee of the Jiangsu Province Academy of Traditional Chinese Medicine (animal ethical code authorization number: AEWC-20180510-30) and maintained following the guidelines of the China Council on Animal Management for the care and use of animals. Male C57BL/6J mice ( $\mathrm{n}=30$, 6 weeks of age, weighing 18-20 g) were purchased from Shanghai SLAC Laboratory Animal, China. The mice were housed in a specific pathogen-free facility of the Jiangsu Province Academy of Traditional Chinese Medicine, with 12-h light/dark cycles and free access to water.

The mice were randomized and fed with either standard diet (STD) (control group, STD, 9.35\% kcal from fat, $n=10)$ or with HFD (60\% kcal from fat, $n=20)$ for 8 weeks. HFD mice were further randomized and either fed continually with HFD (HFD group, $n=7$ ) or with calorie restrictive HFD (HFDCR group, reduced the mean amount of HFD by $25 \%$ ). 


\section{Body weight and testicular coefficient}

The body weights of the mice were measured weekly and fasting blood glucose (FBG) was detected every 2 weeks. After the 12-week intervention, all mice were fasted for 8 hours and euthanized via an intraperitoneal injection of $3 \%$ pentobarbital sodium $(100 \mathrm{mg} / \mathrm{kg})$. The bilateral testes of the male mice were excised and weighed, and then immediately frozen in liquid nitrogen and stored at $-80{ }^{\circ} \mathrm{C}$ for other use. The testicular coefficient was calculated using the following formula: (weight of both testes/body weight) $\times 100$.

\section{Testosterone and metabolic features}

At the end of the study period, blood samples obtained from the orbital fossa were centrifuged at normal temperature and $1,500 \mathrm{~g}$ for $20 \mathrm{~min}$, and the serum was collected and stored at $-80{ }^{\circ} \mathrm{C}$. Blood glucose was measured using a commercial glucometer (Roche, USA). The levels of testosterone, triglyceride (TG), total cholesterol (TC), and insulin were measured using enzyme-linked immunosorbent assay (ELISA) kits. Insulin resistance (IR) was calculated using the homeostasis model assessment of IR (HOMA-IR) according to the following formula: fasting insulin $(\mathrm{mmol} / \mathrm{L})$ $\times$ FBG $(\mathrm{mIU} / \mathrm{L}) / 22.5$.

\section{Sperm analysis}

The epididymides were isolated from mice in each group after the 12-week intervention, and the adhering tissues were cleared. They were subsequently minced into $1 \mathrm{~mm}$ pieces and incubated in $2 \mathrm{~mL}$ phosphate-buffered saline buffer solution at $35^{\circ} \mathrm{C}$ for $1 \mathrm{~h}$. The sperm count, motility, and normality were detected by an automatic sperm quality analyzer (SQA-V), which was purchased from Medical Electronic Systems Ltd. (Israel).

\section{Hematoxylin and eosin (HE) staining}

The testes were fixed overnight at $4{ }^{\circ} \mathrm{C}$ in Bouin's fluid, and then paraffin-embedded, sectioned at $5 \mu \mathrm{m}$, and stained with HE for examination under a light microscope (Olympus, Japan).

\section{Terminal deoxynucleotidyl transferase dUTP nick end labeling (TUNEL) assay}

TUNEL was performed to measure testicular apoptosis using the TUNEL assay kit (Roche, Germany). The paraffin-embedded testicular tissues were sectioned at $5 \mu \mathrm{m}$ and put on the shandon (Leica, HI1220) at $37^{\circ} \mathrm{C} 18$ hours, and were subsequently dewaxed and rehydrated, and added DNase-free Proteinase $\mathrm{K}$ for 30 minutes at $37^{\circ} \mathrm{C}$. Then TUNEL reaction mixture was incubated with the tissue sections at $37^{\circ} \mathrm{C}$ for $2 \mathrm{~h}$. After adding 4',6-diamidino2 -phenylindole (DAPI) nuclear stain in mounting medium, positively labeled nuclei (apoptotic cells) were stained green by the fluorescence microscope (Olympus, Japan).

\section{Biochemical analysis}

Ten percent testicular tissue homogenate was prepared on ice, and was then centrifuged at $3,000 \mathrm{~g}$ for $30 \mathrm{~min}$ and $4^{\circ} \mathrm{C}$. The total protein concentration of testicular tissues were detected using the Bradford method. The activity of SOD, GSH-PX, and the concentration of MDA were detected according to the instructions of the kit.

\section{Western blot assay}

Tissues were lysed using RIPA buffer [50 mM Tris $\mathrm{pH}$ 7.4, $150 \mathrm{mM} \mathrm{NaCl}, 1 \% \mathrm{NP}-40,0.5 \%$ sodium deoxycholate, $0.1 \%$ sodium dodecyl sulfate (SDS), and sodium orthovanadate, sodium fluoride, ethylenediaminetetraacetic acid (EDTA), leupeptin] containing protease inhibitors. The tissue lysate was then centrifuged at $12,000 \mathrm{~g}$ and $4{ }^{\circ} \mathrm{C}$ for $30 \mathrm{~min}$ to pellet debris, and the protein concentration of the supernatant was estimated using a BCA Kit. Proteins were then separated using sodium dodecyl sulfate polyacrylamide gel electrophoresis (SDS-PAGE) and transferred to a polyvinylidene fluoride (PVDF) membrane. The membrane was incubated overnight at $4{ }^{\circ} \mathrm{C}$ with primary antibodies specific SIRT1 $(1: 1,000)$ and $\beta$-actin $(1: 6,000)$, followed by incubation with secondary antibodies $(1: 5,000)$. Enhanced chemiluminescence reagents were used for detection, and images were captured via film cassette (Tanon, China) exposure.

\section{Statistical analysis}

Data were expressed as means \pm standard error of the mean (SEM). The difference among groups was determined by one-way analysis of variance and post-hoc Dunnett's $t$-test using Statistical Product and Service Solutions 22.0 (USA). $\mathrm{P}<0.05$ was considered statistically significant. 
Table 1 CR reduced the body weight and increased the testicular coefficient in obese male mice

\begin{tabular}{lcrc}
\hline Groups & \multicolumn{1}{c}{ STD } & HFD & HFDCR \\
\hline Body weight $(\mathrm{g})$ & $32.82 \pm 1.04$ & $45.25 \pm 2.14^{\star}$ & $34.02 \pm 2.11^{\#}$ \\
Testicular coefficient $(\%)$ & $1.62 \pm 0.03$ & $1.31 \pm 0.10^{\star}$ & $1.66 \pm 0.09^{\#}$ \\
\hline
\end{tabular}

Data are shown as means $\pm \mathrm{SEM} ; \mathrm{n}=5-6$ per group $\left({ }^{*}, \mathrm{P}<0.05\right.$ vs. STD group; ${ }^{*}, \mathrm{P}<0.05$ vs. HFD group). CR, calorie restriction; STD, standard diet; HFD, high-fat diet; HFDCR, high-fat diet with calorie restriction; SEM, standard error of the mean.

Table 2 CR improved glucose and lipid metabolism in obese male mice

\begin{tabular}{lccc}
\hline Groups & STD & HFD & HFDCR \\
\hline FBG $(\mathrm{mmol} / \mathrm{L})$ & $6.48 \pm 1.26$ & $13.44 \pm 1.59^{\star}$ & $8.78 \pm 0.63^{\#}$ \\
Insulin $(\mathrm{ng} / \mathrm{mL})$ & $0.36 \pm 0.19$ & $1.42 \pm 0.49^{\star}$ & $0.55 \pm 0.07^{\#}$ \\
HOMA-IR & $2.61 \pm 0.12$ & $18.07 \pm 6.86^{\star}$ & $3.89 \pm 0.36^{\#}$ \\
TG $(\mathrm{mmol} / \mathrm{L})$ & $1.80 \pm 0.12$ & $2.46 \pm 0.13^{\star}$ & $1.95 \pm 0.04^{\#}$ \\
TC $(\mathrm{mmol} / \mathrm{L})$ & $3.67 \pm 0.18$ & $8.69 \pm 0.55^{*}$ & $5.84 \pm 0.33^{\#}$ \\
\hline
\end{tabular}

Data are shown as means $\pm \mathrm{SEM} ; \mathrm{n}=3-5$ per group $\left({ }^{*}, \mathrm{P}<0.05\right.$ vs. STD group; ${ }^{\prime}, \mathrm{P}<0.05$ vs. HFD group). CR, calorie restriction; STD, standard diet; HFD, high-fat diet; HFDCR, high-fat diet with calorie restriction; FBG, fasting blood glucose; HOMA-IR, homeostasis model assessment of insulin resistance; TG, triglyceride; TC, total cholesterol; SEM, standard error of the mean.

\section{Results}

\section{CR reduced the body weight and increased the testicular} coefficient in obese male mice

HFD induced a significant increase in the body weight of mice. The body weight of the HFDCR decreased significantly compared with the HFD. Compared with the STD group, the testicular coefficient of the HFD group was lower, which reversed by CR (Table 1).

\section{CR improved glucose and lipid metabolism in obese male mice}

HFD induced a significant increase in the FBG, insulin, HOMA-IR, TG, and TC in mice. CR markedly reduced the serum level of FBG and improved insulin sensitivity. Moreover, CR also reduced the levels of TG and TC compared with the HFD group (Table 2).

\section{CR improved the serum testosterone in obese male mice}

Compared to the STD group, HFD feeding significantly decreased the serum testosterone level, whereas serum follicle-stimulating hormone (FSH) and luteinizing hormone (LH) levels exhibited a significantly increasing trend. At the same time, compared with the HFD group, the serum testosterone level was significantly increased by CR (Table 3).

\section{CR improved semen quality in obese male mice}

Compared to the STD group, the sperm counts were significantly decreased in the HFD group, which was mitigated in the CR-treated mice. Similarly, the sperm motility and normality in the HFDCR group were higher than that in the HFD group (Table 4).

\section{CR ameliorated the testicular morphological damage in obese male mice}

The testicular morphological analysis showed that the spermatogenic epithelium in STD group mice was regularly arranged with intact basement membranes and abundant spermatogenic cells. Compared with the STD group, the testicular tissue of HFD mice showed obvious lipid droplet infiltration, as well as loss and derangement of spermatogenic cells, a thinner spermatogenic epithelium, and a reduced number of mature sperm. However, the testes of CR mice had organized spermatogenic epithelium with abundant spermatogenic cells, and increased mature sperm (Figure 1). 
Table 3 CR increased testosterone levels in obese male mice

\begin{tabular}{lrrr}
\hline Groups & \multicolumn{1}{c}{ STD } & HFD & HFDCR \\
\hline Testosterone $(\mathrm{ng} / \mathrm{mL})$ & $2.12 \pm 0.21$ & $1.05 \pm 0.45^{*}$ & $3.63 \pm 1.07^{\#}$ \\
LH $(\mathrm{mlU} / \mathrm{mL})$ & $39.00 \pm 4.25$ & $59.28 \pm 4.03^{\star}$ & $41.10 \pm 5.00^{\#}$ \\
FSH $(\mathrm{mlU} / \mathrm{mL})$ & $16.92 \pm 4.23$ & $34.36 \pm 6.26^{*}$ & $16.04 \pm 6.66^{\#}$ \\
\hline
\end{tabular}

Data are shown as means $\pm \mathrm{SEM} ; \mathrm{n}=3-5$ per group ( ${ }^{*}, \mathrm{P}<0.05$ vs. STD group; ${ }^{*}, \mathrm{P}<0.05$ vs. HFD group). CR, calorie restriction; STD, standard diet; HFD, high-fat diet; HFDCR, high-fat diet with calorie restriction; LH, luteinizing hormone; FSH, follicle-stimulating hormone; SEM, standard error of the mean.

Table 4 CR restored sperm quality in obese male mice

\begin{tabular}{lccc}
\hline Groups & STD & HFD & HFDCR \\
\hline Sperm counts $\left(\times 10^{6} \mathrm{~mL}^{-1}\right)$ & $47.88 \pm 9.07$ & $34.26 \pm 9.19^{*}$ & $49.24 \pm 7.30^{\#}$ \\
Sperm motility (\%) & $64.20 \pm 4.60$ & $50.60 \pm 5.41^{*}$ & $66.00 \pm 9.83^{\#}$ \\
Sperm normality (\%) & $10.75 \pm 0.95$ & $7.8 \pm 1.48^{*}$ & $9.6 \pm 1.14^{\#}$ \\
\hline
\end{tabular}

Data are shown as means \pm SEM; $n=4-5$ per group ( ${ }^{*}, \mathrm{P}<0.05$ vs. STD group; ${ }^{*}, \mathrm{P}<0.05$ vs. HFD group). CR, calorie restriction; STD, standard diet; HFD, high-fat diet; HFDCR, high-fat diet with calorie restriction; SEM, standard error of the mean.

\section{CR suppresses HFD-induced apoptosis in obese male mice}

Compared to the STD group, the number of apoptotic cells was significantly increased in the HFD group. However, the number apoptotic cells decreased markedly in HFDCR group (Figure 2).

\section{CR attenuated the oxidative stress level in obese male mice}

Our results showed that SOD activity in HFD mice were decreased compared with the STD group, which could be reversed by CR. Similar trends were observed for GSHPX activity in testicular tissue. Moreover, compared to the STD mice, the production of MDA was increased in HFD group, which was significantly decreased by CR. Hence, CR alleviated the HFD-induced excess oxidative stress level in the testicular tissue of mice (Table 5).

\section{CR inhibited HFD-induced oxidative stress via activation of the SIRT1 patbway}

The expression levels of SIRT1 in testicular tissue were significantly lower in the HFD group than in the STD group $(\mathrm{P}<0.05)$, but were significantly increased by $\mathrm{CR}$ $(\mathrm{P}<0.05$, Figure 3).

\section{Discussion}

The present study showed that long-term moderate CR decreased body weight gain, and the levels of serum TG and TC, as well as IR in mice with HFD-induced obesity. Moreover, CR increased the testicular coefficients, levels of serum testosterone, sperm counts and motility, and reduced the sperm normality in obese mice. Furthermore, CR ameliorated the testicular morphological damage, lipid deposition, and cells apoptosis in obese mice. Finally, we found that CR attenuated the oxidative stress level and increased the protein expressions of SIRT1 in the testicular tissues of obese mice. Taken together, long-term moderate CR improvement of reproductive function may be related to the increased activity of SIRT1 and attenuated oxidative stress reducing lipotoxicity in the testes.

In this study, we found that CR ameliorated HFDinduced obesity. Therefore, interventions targeting the early stage are particularly important to prevent obesity by reducing calorie intake. Moreover, the gestational diabetes mellitus (GDM) is a high risk factor for GDM offspring to develop IR, while CR could ameliorate this adverse outcome (18). Animal models have shown that combined intrauterine and early postnatal CR significantly improve some sequelae in adulthood (19). However, Torrens et al. found that CR 
STD

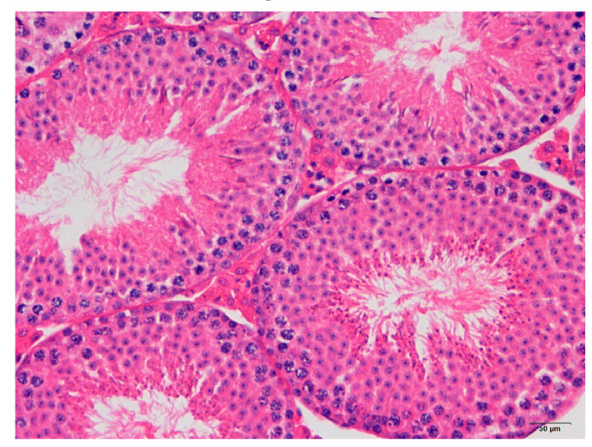

HFD

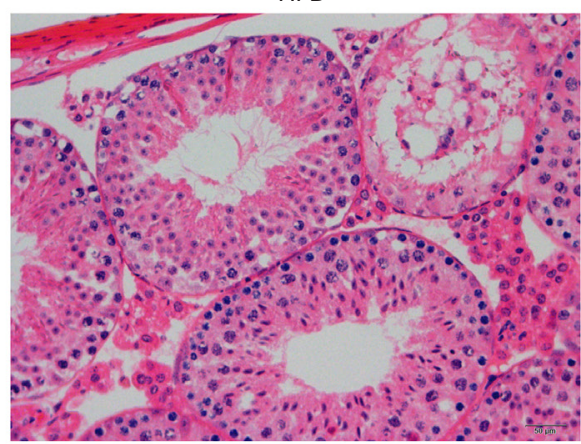

HFDCR

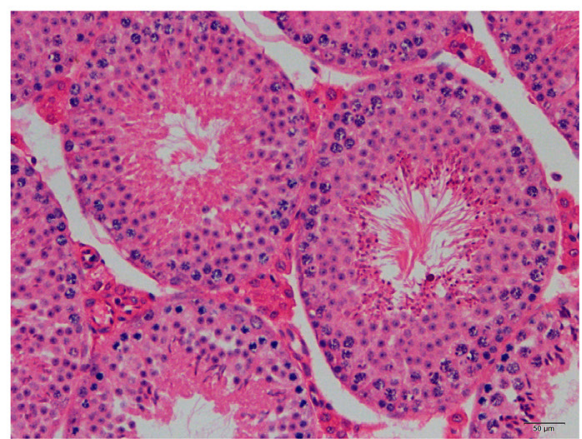

Figure $1 \mathrm{CR}$ ameliorated the testicular morphological damage in obese male mice. HE-stained testicular tissue (200× magnification). CR, calorie restriction; HE, hematoxylin and eosin; STD, standard diet; HFD, high-fat diet; HFDCR, high-fat diet with calorie restriction.

\section{A}
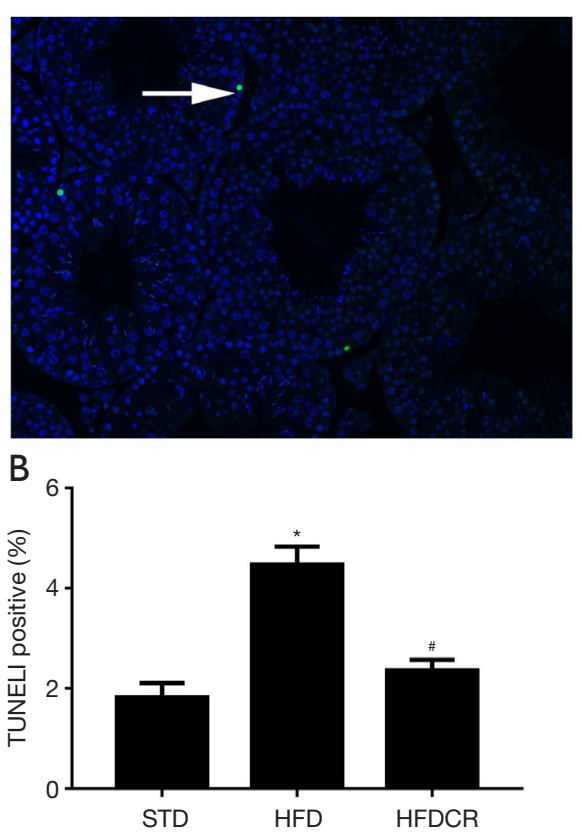

HFD

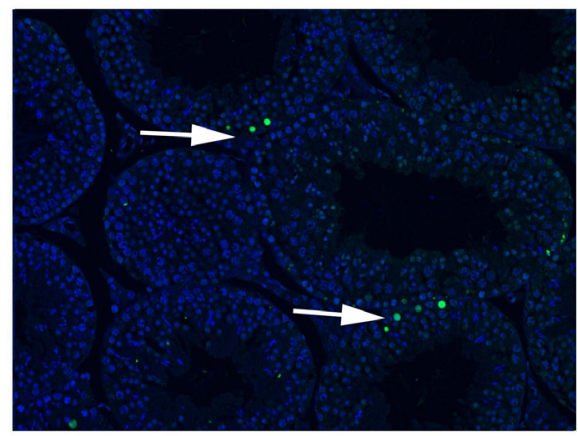

HFDCR

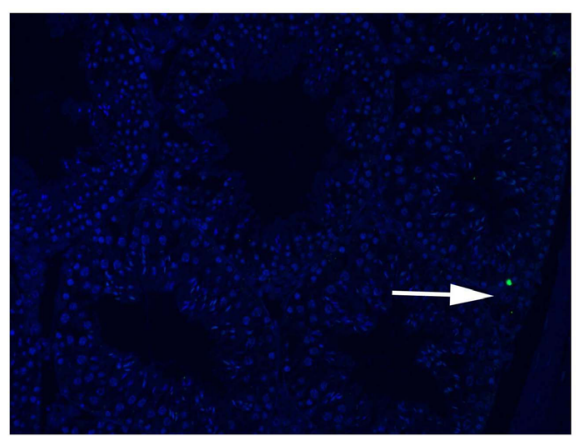

Figure 2 CR suppressed HFD-induced apoptosis in obese male mice. (A) Fluorescent images of testis following double staining for TUNEL (green) and DAPI (blue) (magnification $\times 200$ ). (B) The percentage of TUNEL-positive testicular cells. Data are represented as means \pm SEM for $\mathrm{n}=3$ per group (*, $\mathrm{P}<0.05$ vs. STD group; ${ }^{2}, \mathrm{P}<0.05$ vs. HFD group; arrows indicate apoptotic cells). CR, calorie restriction; HFD, high-fat diet; TUNEL, terminal deoxynucleotidyl transferase dUTP nick end labeling; DAPI, 4',6-diamidino-2-phenylindole; SEM, standard error of the mean; STD, standard diet; HFDCR, high-fat diet with calorie restriction.

intervention in pregnant rats, which induced the offspring had higher HOMA-IR, adipocyte diameter and hepatic TG accumulation than controls under HFD feeding during the age of 4 to 6 months (20). Hence, CR had a positive effects on improving metabolic disturbance, but an opposite effect on the offspring of normal pregnant animals due to the maternal undernutrition during gestation.

It is well known that obesity is associated with male infertility. However, the question remains as to whether moderate $\mathrm{CR}$ can reverse the damage to male reproduction caused by obesity. A previous study reported that CR did not affect testosterone levels and reproduction in mutant 
Table 5 CR attenuated the oxidative stress level in obese male mice

\begin{tabular}{lcrr}
\hline Groups & STD & HFD & HFDCR \\
\hline SOD (U/mg protein) & $266.82 \pm 12.56$ & $225.18 \pm 11.04^{*}$ & $255.25 \pm 12.43^{\#}$ \\
MDA (nmol/mg protein) & $0.65 \pm 0.04$ & $1.02 \pm 0.11^{*}$ & $0.70 \pm 0.15^{\#}$ \\
GSH-PX (U/mg protein) & $197.49 \pm 17.84$ & $141.80 \pm 10.50^{*}$ & $175.05 \pm 12.53^{\#}$ \\
\hline
\end{tabular}

Data are shown as means $\pm \mathrm{SEM} ; \mathrm{n}=6$ per group $\left(^{*}, \mathrm{P}<0.05\right.$ vs. STD group; ${ }^{*}, \mathrm{P}<0.05$ vs. HFD group). CR, calorie restriction; $\mathrm{STD}$, standard diet; HFD, high-fat diet; HFDCR, high-fat diet with calorie restriction; SOD, superoxide dismutase; MDA, malondialdehyde; GSH-PX, glutathione peroxidase; SEM, standard error of the mean.
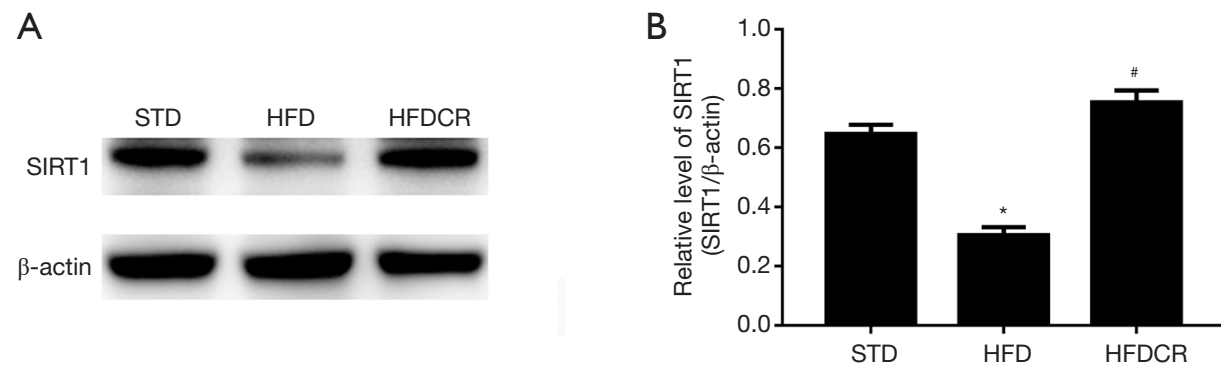

Figure $3 \mathrm{CR}$ increased SIRT1 activity in the testicular tissue of obese male mice. (A,B) Protein expression levels of SIRT1. Data are represented as means $\pm \mathrm{SEM} ; \mathrm{n}=3$ per group $\left({ }^{*}, \mathrm{P}<0.05\right.$ vs. STD group; ${ }^{*}, \mathrm{P}<0.05$ vs. HFD group). CR, calorie restriction; SIRT1, NADdependent deacetylase sirtuin-1; SEM, standard error of the mean; HFD, high-fat diet; STD, standard diet; HFDCR, high-fat diet with calorie restriction.

mice (21). Moreover, CR was shown to delay reproductive development and induced negative energy balance in prepubertal rats (22). However, we found that CR improved reproductive function in obese mice, which are inconsistent with the results of the aforementioned previous studies. Notably, these previous studies used animals with normal lipid and glucose metabolism. In this study, the effect of CR was analyzed during adulthood in obese mice, which may explain the discrepancy in the findings.

Our data showed the level of serum testosterone in the CR group was elevated, compared to that in the HFD group. In general, the hypothalamus produces and releases gonadotropin-releasing hormone, which stimulates the production of FSH and LH in the anterior pituitary. FSH and LH act on the testicles to stimulate spermatogenesis and testosterone, respectively. We found that the level of serum testosterone decreased in HFD mice, but not the levels of FSH and LH. The changes in sex hormones may be related to the negative feedback regulation of the hypothalamus. Testosterone deficiency is associated with energy imbalance, impaired glucose control, reduced insulin sensitivity, and dyslipidemia (23). Therefore, the level of serum testosterone remained at a high level even after a long period of moderate CR intervention, which may be related to the improvement of serum lipid, glucose, and IR in obese male mice.

Moreover, apart from dyslipidemia in HFD-induced obese male mice, lipid accumulation was abundant and the structure of the testicular tissue was destroyed. This result demonstrated that the testes were in a lipotoxic microenvironment. We subsequently found that CR markedly reduced the lipid deposition, morphological damage, and cell apoptosis in the testes. It has been reported that excessive fat causes lipotoxic effects, which is mediated by oxidative stress (24). Specifically, testicular lipids and proteins are highly sensitive to oxygen free radical attacks. Sperm is particularly vulnerable to reactive oxygen species (ROS). Excessive ROS attacks polyunsaturated fatty acids on the sperm membrane, causing lipid peroxidation, thereby damaging the sperm membrane structure, which affects sperm motility ultimately leads to male infertility $(8,25)$.

The present results showed that CR increased the activity SOD and GSH-PX, and decreased the MDA level in the testes of HFD-induced obese male mice. Under normal conditions, oxidative damage and antioxidant defense 
systems are in a state of dynamic balance in cells. HFD feeding destroyed the balance and resulted in oxidative stress, which was related to excessive production of ROS. It is well known that the clean systems of ROS include SOD, GSH-PX, catalase and the peroxidase family in the body. The changes in the content and activity of the enzymes, which directly affect the intracellular ROS content. SOD and GSH-PX are important and indispensable in the entire defense strategy of antioxidants. The vitality of SOD indirectly reflects the ability to scavenge oxygen free radicals $(26,27)$. MDA is the last product of lipid peroxidation, which is a significant biomarker of oxidative stress (28). Hence, it is possible that CR may mitigated HFD-induced sperm abnormality, and increased sperm count and motility, by attenuating oxidative stress.

Furthermore, we found that CR increased the protein expressions of SIRT1 in testicular tissues of HFD-induced obese male mice. SIRT1 is a class-III histone deacetylase, a highly conserved $\mathrm{NAD}^{+}$-dependent lysine deacetylase, which is deeply involved in gene regulation, genome stability maintenance, apoptosis, autophagy, senescence, proliferation, aging, and tumorigenesis (29). Transgenic models provide strong evidence that SIRT1 is involved in spermatogenesis by influencing the specific functions of male germ cells, Sertoli cells, and Leydig cells (30). A previous study showed that SIRT1 degradation induces IR via lipotoxicity (31). Moreover, SIRT1 activation has been shown to attenuate the levels of oxidative stress (it decreased MDA levels, and increased SOD and GSH-PX levels), which suggests that it protected the cells against ROS-induced damage (32). A recent study showed that the activation of SIRT1 can alleviate oxidative stress, DNA damage, and protect the testes from lipotoxicity (3). It has been also reported that SIRT1 acts as a central mediator of the cellular responses to CR (33). CR protected the ageing kidney include increasing SIRT1 levels, AMP-activated protein kinase and autophagy, reducing mammalian target of rapamycin, inflammation and oxidative stress (34). Therefore, we speculate that CR protects mouse testes from HFD induced-lipotoxicity by reducing oxidative stress, probably via activation of SIRT1 signaling.

The limitations of the present study should be noted. Firstly, we did not perform experiments for verification, such as SIRT1 deficiency, to determine the role of SIRT1 in the CR-related therapeutic effects in vivo and in vitro. Secondly, we did not measure the levels of SIRT1 and oxidative stress in the different cells in testis, such as germ cells, Sertoli cells, and Leydig cells. Thirdly, the current study did not test testicular cholesterol and free fatty acids level to evaluate the beneficial effect of CR on lipotoxicity.

In conclusion, our results indicate that long-term moderate CR protected the testicular histology and semen quality that was disrupted in HFD-induced obese mice via increasing SIRT1 activity and enhancement of antioxidative enzymes in the testes.

\section{Acknowledgments}

We received considerable technical assistance for this study from Yijiao Xu, Xingjia Li, and Xiaodong Mao.

Funding: We acknowledge funding from Six Talent Peaks Project in Jiangsu Province (WSN-035); National Nature Science Foundation of China (No. 81800756); and the Research Project of Cadre Health Care in Jiangsu Province (BJ17029).

\section{Footnote}

Reporting Checklist: The authors have completed the ARRIVE reporting checklist. Available at http://dx.doi. org/10.21037/atm-21-2458

Data Sharing Statement: Available at http://dx.doi. org/10.21037/atm-21-2458

Conflicts of Interest: All authors have completed the ICMJE uniform disclosure form (available at http://dx.doi. org/10.21037/atm-21-2458). All authors report funding from Six Talent Peaks Project in Jiangsu Province (WSN035); National Nature Science Foundation of China (No. 81800756); and the Research Project of Cadre Health Care in Jiangsu Province (BJ17029). The authors have no other conflicts of interest to declare.

Etbical Statement: The authors are accountable for all aspects of the work in ensuring that questions related to the accuracy or integrity of any part of the work are appropriately investigated and resolved. All animal studies were approved by the Animal Experimentation Ethics Committee of the Jiangsu Province Academy of Traditional Chinese Medicine (Animal ethical code authorization number: AEWC-20180510-30) and maintained following the guidelines of the China Council on Animal Management for the care and use of animals.

Open Access Statement: This is an Open Access article 
distributed in accordance with the Creative Commons Attribution-NonCommercial-NoDerivs 4.0 International License (CC BY-NC-ND 4.0), which permits the noncommercial replication and distribution of the article with the strict proviso that no changes or edits are made and the original work is properly cited (including links to both the formal publication through the relevant DOI and the license). See: https://creativecommons.org/licenses/by-nc-nd/4.0/.

\section{References}

1. NCD Risk Factor Collaboration (NCD-RisC). Trends in adult body-mass index in 200 countries from 1975 to 2014: a pooled analysis of 1698 population-based measurement studies with 19.2 million participants. Lancet 2016;387:1377-96. Erratum in: Lancet 2016;387:1998.

2. Bastien M, Poirier P, Lemieux I, et al. Overview of epidemiology and contribution of obesity to cardiovascular disease. Prog Cardiovasc Dis 2014;56:369-81.

3. Xu D, Liu L, Zhao Y, et al. Melatonin protects mouse testes from palmitic acid-induced lipotoxicity by attenuating oxidative stress and DNA damage in a SIRT1dependent manner. J Pineal Res 2020;69:e12690.

4. Ma J, Wu L, Zhou Y, et al. Association between BMI and semen quality: an observational study of 3966 sperm donors. Hum Reprod 2019;34:155-62.

5. Bieniek JM, Kashanian JA, Deibert CM, et al. Influence of increasing body mass index on semen and reproductive hormonal parameters in a multi-institutional cohort of subfertile men. Fertil Steril 2016;106:1070-5.

6. Ghosh S, Mukherjee S. Testicular germ cell apoptosis and sperm defects in mice upon long-term high fat diet feeding. J Cell Physiol 2018;233:6896-909.

7. Mu Y, Yan WJ, Yin TL, et al. Diet-induced obesity impairs spermatogenesis: a potential role for autophagy. Sci Rep 2017;7:43475.

8. Bisht S, Faiq $M$, Tolahunase $M$, et al. Oxidative stress and male infertility. Nat Rev Urol 2017;14:470-85.

9. Dupont C, Faure C, Sermondade N, et al. Obesity leads to higher risk of sperm DNA damage in infertile patients. Asian J Androl 2013;15:622-5.

10. Yi X, Tang D, Cao S, et al. Effect of different exercise loads on testicular oxidative stress and reproductive function in obese male mice. Oxid Med Cell Longev 2020;2020:3071658.

11. Rada P, Pardo V, Mobasher MA, et al. SIRT1 controls acetaminophen hepatotoxicity by modulating inflammation and oxidative stress. Antioxid Redox
Signal 2018;28:1187-208

12. Seifert EL, Caron AZ, Morin K, et al. SirT1 catalytic activity is required for male fertility and metabolic homeostasis in mice. FASEB J 2012;26:555-66.

13. Most J, Tosti V, Redman LM, et al. Calorie restriction in humans: an update. Ageing Res Rev 2017;39:36-45.

14. Nair PM, Khawale PG. Role of therapeutic fasting in women's health: An overview. J Midlife Health 2016;7:61-4.

15. Best D, Avenell A, Bhattacharya S. How effective are weight-loss interventions for improving fertility in women and men who are overweight or obese? A systematic review and meta-analysis of the evidence. Hum Reprod Update 2017;23:681-705.

16. Samavat J, Cantini G, Lotti F, et al. Massive weight loss obtained by bariatric surgery affects semen quality in morbid male obesity: a preliminary prospective doublearmed study. Obes Surg 2018;28:69-76.

17. Schulte DM, Hahn M, Oberhäuser F, et al. Caloric restriction increases serum testosterone concentrations in obese male subjects by two distinct mechanisms. Horm Metab Res 2014;46:283-6.

18. Li T, Chen K, Liu G, et al. Calorie restriction prevents the development of insulin resistance and impaired lipid metabolism in gestational diabetes offspring. Pediatr Res 2017;81:663-71.

19. Redman LM, Smith SR, Burton JH, et al. Metabolic slowing and reduced oxidative damage with sustained caloric restriction support the rate of living and oxidative damage theories of aging. Cell Metab 2018;27:805-15.e4.

20. Torrens JM, Orellana-Gavaldà JM, Palou M, et al. Enhancing hepatic fatty acid oxidation as a strategy for reversing metabolic disorders programmed by maternal undernutrition during gestation. Cell Physiol Biochem 2014;33:1498-515.

21. Rocha JS, Bonkowski MS, França LR, et al. Mild calorie restriction does not affect testosterone levels and testicular gene expression in mutant mice. Exp Biol Med (Maywood) 2007;232:1050-63.

22. Rizzoto G, Sekhar D, Thundathil JC, et al. Calorie restriction modulates reproductive development and energy balance in pre-pubertal male rats. Nutrients 2019;11:1993.

23. Kelly DM, Jones TH. Testosterone and obesity. Obes Rev 2015;16:581-606.

24. Ye J, Luo D, Xu X, et al. Metformin improves fertility in obese males by alleviating oxidative stress-induced blood-testis barrier damage. Oxid Med Cell Longev 


\section{Page 10 of 10}

2019;2019:9151067.

25. Smits RM, Mackenzie-Proctor R, Yazdani A, et al. Antioxidants for male subfertility. Cochrane Database Syst Rev 2019;3:CD007411.

26. Yu J, Dong HW, Shi LT, et al. Reproductive toxicity of perchlorate in rats. Food Chem Toxicol 2019;128:212-22.

27. Liu S, Yang D, Yu L, et al. Effects of lycopene on skeletal muscle-fiber type and high-fat diet-induced oxidative stress. J Nutr Biochem 2021;87:108523.

28. Adnan MT, Amin MN, Uddin MG, et al. Increased concentration of serum MDA, decreased antioxidants and altered trace elements and macro-minerals are linked to obesity among Bangladeshi population. Diabetes Metab Syndr 2019; 13:933-8.

29. Alves-Fernandes DK, Jasiulionis MG. The role of SIRT1 on DNA damage response and epigenetic alterations in cancer. Int J Mol Sci 2019;20:3153.

30. Tatone C, Di Emidio G, Barbonetti A, et al. Sirtuins in

Cite this article as: Zhang S, Zhang M, Sun S, Wei X, Chen Y, Zhou P, Zheng R, Chen G, Liu C. Moderate calorie restriction ameliorates reproduction via attenuating oxidative stressinduced apoptosis through SIRT1 signaling in obese mice. Ann Transl Med 2021;9(11):933. doi: 10.21037/atm-21-2458

\section{Zhang et al. CR ameliorates reproduction in obese male mice}

gamete biology and reproductive physiology: emerging roles and therapeutic potential in female and male infertility. Hum Reprod Update 2018;24:267-89.

31. Ren X, Chen N, Chen Y, et al. TRB3 stimulates SIRT1 degradation and induces insulin resistance by lipotoxicity via COP1. Exp Cell Res 2019;382:111428.

32. Ren Y, Du C, Shi Y, et al. The Sirt1 activator, SRT1720, attenuates renal fibrosis by inhibiting CTGF and oxidative stress. Int J Mol Med 2017;39:1317-24.

33. Pardo R, Velilla M, Herrero L, et al. Calorie restriction and SIRT1 overexpression induce different gene expression profiles in white adipose tissue in association with metabolic improvement. Mol Nutr Food Res 2021;65:e2000672.

34. Wang SY, Cai GY, Chen XM. Energy restriction in renal protection. Br J Nutr 2018;120:1149-58.

(English Language Editor: A. Kassem) 